\title{
ERRATUM
}

\section{Ubiquity of Putative Type III Secretion Genes among Clinical and Environmental Burkholderia pseudomallei Isolates in Northern Australia}

\author{
H. C. Smith-Vaughan, D. Gal, P. M. Lawrie, C. Winstanley, K. S. Sriprakash, \\ and B. J. Currie \\ University of Queensland and Queensland Institute of Medical Research, Brisbane, and Menzies School of Health \\ Research, Royal Darwin Hospital, Darwin, Australia, and Department of Medical Microbiology and \\ Genitourinary Medicine, University of Liverpool, Liverpool, United Kingdom
}

Volume 41, no. 2, p. 883-885, 2003. Page 883, abstract, line 1: "Horseradish peroxidase-like type III secretion genes" should read "Type III secretion (TTS1) genes of an HRP (hypersensitivity response and pathogenicity)-like locus."

Page 883, column 2, lines 10 and 11: "a horseradish peroxidase (hypersensitivity response and pathogenicity) locus" should read "an HRP (hypersensitivity response and pathogenicity) locus." 